\title{
BRCA1: linking HOX to breast cancer suppression
}

Kideok Jin and Saraswati Sukumar*

\begin{abstract}
Homeobox (HOX) genes play key roles in embryogenesis and tissue differentiation. Recently, a number of groups have reported altered HOX gene expression in breast cancer. However, the mechanism of HOX gene regulation and the search for direct targets of its transcriptional regulatory function have been minimally fruitful. Recently, Gilbert and colleagues reported that HOXA9 restrains breast cancer progression by upregulation of $B R C A 1$, a tumor suppressor. This finding raises our hope that more, rather elusive targets of HOX genes important in tumor progression or suppression will be found in the future.
\end{abstract}

\section{Background}

Homeobox $(H O X)$ genes, a family of transcription factors, function as key determinants of anterio-posterior patterning of animal embryos and the development of several organs [1]. Formed by gene duplication, 39 members of the $H O X$ gene family are arranged in four $H O X$ clusters, A, B, C, and D on human chromosomes 7 , 17, 12, and 2, respectively. Altered HOX gene expression has been implicated in differentiation, invasion, epithelialmesenchymal transition, apoptosis, and receptor signaling in a variety of cancer types [2-8]. In breast cancer, some $H O X$ genes are overexpressed whereas others are underexpressed, and mutations are rarely observed [9]. However, the molecular mechanism of HOX gene action has remained elusive. Ten years after a study by Raman and colleagues [4] demonstrated that HOXA5 can control $p 53$ transcription and that loss of HOXA5 correlated with loss of p53 expression in breast cancer, a study by Gilbert and colleagues [10] has pinpointed another gene critical for breast cell function, $B R C A 1$, as the target of a $H O X$ gene. In the latter study, the authors

*Correspondence: saras@jhmi.edu

Breast Cancer Program, Sidney Kimmel Comprehensive Cancer Center at Johns Hopkins, Johns Hopkins University School of Medicine, 1650 Orleans Street, Room 143 CRB1, Baltimore, MD, 21231, USA present strong evidence that HOXA9 inhibits breast cancer progression by modulating expression of BRCA1.

\section{The paper}

HOXA9 as a breast cancer-related gene piqued the interest of this group when an Affymetrix microarray analysis (Affymetrix, Santa Clara, CA, USA) of five paired sets of microdissected breast tumors and their adjacent normal tissue identified HOXA9 as 1 of 115 transcripts that showed tumor-specific downregulation. Examination of publicly available databases showed that HOXA9 is significantly downregulated in breast cancer and correlated with disease aggressiveness. The authors then went on to investigate the effects of HOXA9 reexpression on tumor cell proliferation, survival, and differentiation. Interestingly, overexpression of HOXA9 in estrogen receptor-negative cell lines inhibited breast cancer cells through decreases of cell growth, survival, and invasiveness and changes in morphogenesis. In addition, to find targets of HOXA9, the authors performed global transcriptional profiling of HOXA9overexpressing MDA-MD-231 cells. They found that the expression of BRCA1, the breast cancer susceptibility gene and a well-known tumor suppressor gene, was higher in HOXB9-overexpressing cells compared with vector control cells. By performing chromatin immunoprecipitation analysis, they found that HOXA9 directly binds to the $5^{\prime}$ promoter region of BRCA1. If HOXA9 is a regulator of tumor suppressor genes, they reasoned, loss of HOXA9 in nonmalignant MCF-10A mammary epithelial cells should lead to malignant transformation. To test this concept, the authors depleted HOXA9 in MCF10A cells with short hairpin RNAs (shRNAs). This led to increases of cell growth, invasiveness, and survival phenotype. Similar changes were seen upon knockdown of BRCA1. Co-expression of HOXA9 with mutants of BRCA1 reduced the ability of HOXA9 to inhibit tumorigenesis of breast cancer cells. In addition, by immunohistochemistry studies, the authors provide clinical evidence that there is a strong positive association between expression of HOXA9 and that of BRCA1. In summary, Gilbert and colleagues [10] demonstrated that HOXA9 inhibits cell proliferation and survival of human breast cancer cells, possibly by upregulation of BRCA1. These findings provide an explanation for the 
concomitant loss of HOXA9 and BRCA1 expression in breast cancer.

\section{Viewpoint}

Previous studies have shown that HOXA9 is highly methylated and downregulated in several types of cancer, including breast cancer, but the consequences of this loss were not explored [11]. The article by Gilbert and colleagues [10] clarifies, for the first time, the clinical correlation and relevance of loss of HOXA9 and breast tumorigenesis. Second, the demonstration that shRNAmediated knockdown of HOXA9 promotes tumorigenesis in nonmalignant mammary epithelial cells supports the inference that, in normal cells, HOXA9 inhibits cell proliferation and survival of human breast cancer cells, possibly by upregulation of BRCA1. However, many questions remain. Can the loss of the HOXA9 gene alone disrupt homeostasis in breast cells and initiate/promote tumorigenesis? Do our experiments in tissue culture faithfully reflect in vivo events? In 1999, Chen and Capecchi [12] reported mild to no phenotypic changes in mammary glands of HOXA9/HOXB9/HOXD9 knockout mice; these triple-knockout female mice showed hypoplasia of the mammary glands but only upon induction of pregnancy and lactation; however, they did not develop tumors. In the article of Gilbert and colleagues [10], the arrayed tumor samples were from five individuals ranging in age from 44 to 54 years and were presumably parous. Did parity play a role in heightening the effects of the loss of HOXA9 in their breast cells? Are HOXA9 and BRCA1 also downregulated in nulliparous patients with breast cancer? Why do the other paralogs - HOXB9, C9, or D9 not substitute for the function of the lost HOXA9? Perhaps HOXA9 is the only paralog specifically expressed in breast epithelial cells. Interestingly, as mentioned by the authors, HOXA9 is overexpressed and activated in acute myeloid leukemia and endothelial cells. But these cells do not show concomitantly high BRCA1 expression $[13,14]$, supporting the dogma that HOX target genes are cell- and tissue-specific. In conclusion, the findings in this paper are very informative, establishing the ability of HOXA9 to exert its vigilante function through regulating the expression of BRCA1 in breast cells. The paper raises as many questions as it answers - a hallmark of an interesting paper.

Abbreviations

HOX, homeobox; shRNA, short hairpin RNA.

\section{Competing interests}

The authors declare that they have no competing interests.

Published: 23 July 2010

\section{References}

1. Krumlauf R: Hox genes in vertebrate development. Cell 1994, 78:191-201.

2. Cillo C, Cantile M, Faiella A, Boncinelli E: Homeobox genes in normal and malignant cells. J Cell Physio/ 2001, 188:161-169.

3. Abate-Shen C: Deregulated homeobox gene expression in cancer: cause or consequence? Nat Rev 2002, 2:777-785.

4. Raman V, Martensen SA, Reisman D, Evron E, Odenwald WF, Jaffee E, Marks J, Sukumar S: Compromised HOXA5 function can limit p53 expression in human breast tumours. Nature 2000, 405:974-978.

5. Trivedi CM, Patel RC, Patel CV: Homeobox gene HOXA9 inhibits nuclear factor-kappa B dependent activation of endothelium. Atherosclerosis 2007 195:e50-60.

6. Trivedi CM, Patel RC, Patel CV: Differential regulation of HOXA9 expression by nuclear factor kappa B (NF-kappaB) and HOXA9. Gene 2008, 408:187-195.

7. Wu X, Chen H, Parker B, Rubin E, Zhu T, Lee JS, Argani P, Sukumar S: HOXB7, a homeodomain protein, is overexpressed in breast cancer and confers epithelial-mesenchymal transition. Cancer Res 2006, 66:9527-9534.

8. Daftary GS, Taylor HS: Endocrine regulation of HOX genes. Endocr Rev 2006 27:331-355.

9. Shah N, Sukumar S: The Hox genes and their roles in oncogenesis. Nat Rev Cancer 2010, 10:361-371.

10. Gilbert PM, Mouw JK, Unger MA, Lakins JN, Gbegnon MK, Clemmer VB, Benezra M, Licht JD, Boudreau NJ, Tsai KK, Welm AL, Feldman MD, Weber BL, Weaver VM: HOXA9 regulates BRCA1 expression to modulate human breast tumor phenotype. J Clin Invest 2010, 120:1535-1550.

11. Reynolds PA, Sigaroudinia M, Zardo G, Wilson MB, Benton GM, Miller CJ, Hong C, Fridlyand J, Costello JF, TIsty TD: Tumor suppressor p16INK4A regulates polycomb-mediated DNA hypermethylation in human mammary epithelial cells. J Biol Chem 2006, 281:24790-24802.

12. Chen F, Capecchi MR: Paralogous mouse Hox genes, Hoxa9, Hoxb9, and Hoxd9, function together to control development of the mammary gland in response to pregnancy. Proc Natl Acad Sci U S A 1999, 96:541-546.

13. Look AT: Oncogenic transcription factors in the human acute leukemias. Science 1997, 278:1059-1064.

14. Bruhl T, Urbich C, Aicher D, Acker-Palmer A, Zeiher AM, Dimmeler S: Homeobox A9 transcriptionally regulates the EphB4 receptor to modulate endothelial cell migration and tube formation. Circ Res 2004, 94:743-751.

doi:10.1186/bcr2600

Cite this article as: Jin K, Sukumar S: BRCA1: linking HOX to breast cancer suppression. Breast Cancer Research 2010, 12:306 